\title{
TABULA GRATULATORIA
}

Walter Abel, Berlin

Horst von Abercron, Berlin

Alfred Adam, Bethel

Theodor W. Adorno, Frankfurt

Adam Adrio, Berlin

Kurt Aland, Münster

Heinrich Albertz, Berlin

Gerhard Alexander, Hamburg

Paul Althaus, Erlangen

Ernst-Heinz Amberg, Lcipzig

Georg Anders, Bonn

Wilhelm Andersen, Neuendettelsau

Carl Andresen, Göttingen

Wilhelm Anz, Bethel

Adolf Arndt, Berlin

Robert Bach, Wuppertal

Helmut Bandt, Greifswald

Hans Bardtke, Leipzig

Ernst Barnikol, Halle

Otto Bauernfeind, Tübingen

Friedrich Baumgärtel, Erlangen

Heinrich Benckert, Rostock

Ernst Benz, Marburg

Christian Berg, Berlin

Heinz Bernau, Naumburg

Alfred Berner, Berlin

Karl-Heinz Bernhardt, Rostock

Hans Besch, Glücksburg

Kurt Bittel, Berlin

Georg Biundo, Mainz

Hans-Joachim Bock, Berlin

Rudolf Bohren, Wuppertal

Borngässer, Marburg

Günther Bornkamm, Heidelberg

Heinrich Bornkamm, Heidelberg

Gerd Brand, Berlin

Herbert Braun, Mainz

Edgar Breitenbach, z. Z. München

Gerhard Brennecke, Berlin

Roswitha Brost, Meinsdorf

Walter Bruhns, Schwerin

Peter Brunner, Heidelberg
C. Colpe, Göttingen

Hans Conzelmann, Göttingen

Georg v. Dadelsen, Hamburg

Ernst Dammann, Marburg

Fritz Dehn, Berlin

Friedrich Delekat, Mainz

Walter Delius, Berlin

Christoph Demke, Berlin

Otto Dibelius, Berlin

Hermann Diem, Tübingen

Otto Dilschneider, Berlin

Martin Doernc, Göttingen

Hermann Dörries, Göttingen

Herbert Donner, Göttingen

Walter Dreß, Berlin

Alfred Dürr, Göttingen

Werner Düttmann, Berlin

Gerhard Ebeling, Zürich

Karl-Gottfried Eckart, Berlin

Gcorg Eichholz, Wuppertal

Herbert von Einem, Bonn

Otto Eißfeldt, Halle

Eduard Fllwein, Neuendettelsau

Rudolf Elvers, Berlin

Hans Engelland, Kiel

Fritz T. Epstein, Bad Godesberg

Herta M. Epstein, Bad Godesberg

Erich Fascher, Berlin

Karl-Gustav Fellerer, Köln

Martin Fischer, Berlin

Werner Foerster, Münster

Georg Fohrer, Erlangen

Ferdinand Friedensburg, Berlin

Gerhard Friedrich, Erlangen

Hans-Georg Fritzsche, Berlin

Kurt Frör, Erlangen

Ernst Fuchs, Marburg

Otto-Heinrich v. d. Gablentz, Berlin

Walter Gebhardt, Tübingen

Karl-Heinz Gefaeller, Berlin 
$\mathrm{X}$

Paul Geißler, Augsburg

Walter Gerstenberg, Tübingen

Gerhardt Giese, Berlin

Gerhard Gloege, Bonn

Walter Göbell, Kiel

Erwin Gohrbandt, Berlin

Dietrich Goldschmidt, Berlin

Helmut Gollwitzer, Berlin

Leonhard Goppelt, Hamburg

Heinrich Greeven, Kiel

Will Grohmann, Berlin

Gottfried Grote, Berlin

Karl Grzegorzewski, Bethel

Antonius Gunneweg, Marburg

Wilibald Gurlitt, Freiburg

G. Haendler, Rostock

Otto Haendler, Berlin

Johannes Hamel, Naumburg

Oskar Hammelsbeck, Wuppertal

G. Harbsmeier, Göttingen

Günther Harder, Berlin

Axel von Harnack, Tübingen

Christoph Haufe, Leipzig

Friedrich Haufe, Leipzig

Herbert Hausmann, Berlin

Diethard Hellmann, Mainz

Heinz-Ludwig Hempel, Mainz

Johannes Hempel, Göttingen

Erich Henschel, Berlin

Karl-Heinz Herrmann, Berlin

Franz Hesse, Münster

Eva Heßler, Naumburg

Johannes Erich Heyde, Berlin

Jürgen Heydrich, Berlin

Franz Reinhold Hildebrandt, Berlin

Georg Hoffmann, Kiel

Wilhelm Hoffmann, Stuttgart

Friedrich Hofmann, Nürnberg

Gustav Hofmann, München

Walter Holsten, Mainz

Hildebrecht Hommel, Tübingen

Ernst Hornig, Görlitz

Kurt Ihlenfeld, Berlin

Käthe Iwand, Köln

Gerhard Jacobi, Oldenburg

Paul Jacobs, Münster

Johannes Jänidke, Magdeburg

Werner Jahrmann, Berlin

Wilhelm Jannasch, Mainz
Tabula Gratulatoria

Hans-Hinrich Jenssen, Berlin

Alfred Jepsen, Greifswald

Rudolf Juchhoff, Köln

Eberhard Jüngel, Berlin

Paul Kaegbein, Berlin

Ehrhard Kamlah, Mainz

Heinrich Karpp, Bonn

Hermann Keller, Stuttgart

Alfred Kelletat, Berlin

Konrad Kettig, Stuttgart

Franz Heinrich Kettler, Münster

Ernst Kinder, Münster

Sergius v. Kissel, Berlin

H. Kittel, Münster

Bernhard Klaus, Erlangen

Karl-Heinz Köhler, Berlin

Joachim Konrad, Bonn

Helmut Krämer, Bethel

Heinrich Kraft, Kiel

Hans-Joachim Kraus, Hamburg

Gerhard Krause, Bonn

Walter Kreck, Bonn

Hans von Kress, Berlin

Georg Kretschmar, Hamburg

Werner Krieg, Köln

Herbert Krimm, Heidelberg

Erwin Kroll, Berlin

Harald Kruska, Berlin

Helmut Külz, Berlin

Werner Kümmel, Marburg

Walter Künneth, Erlangen

Karl Kupisch, Berlin

Arnulf Kuschke, Mainz

Clemens Lagemann, Münster

Ernst Lange, Berlin

Thomas-M. Langner, Berlin

Franz Lau, Leipzig

Jan Lauts, Karlsruhe

Paul Lefrancq, Valencinennes

Arno Lehmann, Halle

Peter Lehrecke, Berlin

Fairy v. Lilienfeld, Naumburg

Wilhelm Loew, Mainz

Ilse von Loewenclau, Berlin

Walter von Loewenich, Erlangen

Eduard Lohse, Kiel

Rudolf Lorenz, Mainz

Georg Luck, Bonn

Ulrich Luck, Bethel

Ingetraut Ludolphy, Leipzig 
Hildegard Lullies, Berlin

Otto Luschnat, Berlin

Fritz Maass, Berlin

Barbara Mahrenholz, Hannover

Christhard Mahrenholz, Hannover

Wolf-Dieter Marsch, Wuppertal

Albrecht Martin, Mainz

Willi Marxsen, Münster

Christian Maurer, Bethel

Wilhelm Maurer, Erlangen

Kurt Meier, Leipzig

Walter Menn, Mainz

Hans-Arnold Metzger, Eßlingen

Heinrich Meyer, Lübeck

Ernst-Wilhelm Meyer, Berlin

Manfred Mezger, Mainz.

Otto Michel, Tübingen

Margarete Möller, Naumburg

Ilsabe Moering, Naumburg

Ordo philosophorum Universitatis

Maguntinae nomine Johannis Gutenbergii

Exornatae

Hans Moritz, Lcipzig

Fritz Moser, Berlin

Dedo Müller, Leipzig

Erwin Mülhaupt, Wuppertal

Gerhard Müller, Marburg

Wolfram Müllerburg, Berlin

Wolfgang Müller-Lauter, Berlin

Hans-Rudolf Müller-Schwefe, Hamburg

Hans Nachtsheim, Berlin

William Nagel, Greifswald

Wolfgang Nauck, Wuppertal

Hermann Neubert, Berlin

Fritz Neugebauer, Naumburg

Gottfried Noth, Dresden

Martin Noth, Bonn

Konrad Onasch, Halle

Dietrich v. Oppen, Marburg

Gert Otto, Mainz

Wolfhart Pannenberg, Mainz

Anneliese Passier, Berlin

Ernst Pepping, Berlin

Lothar Perlitt, Berlin

Erhard Peschke, Halle

Edith Picht-Axenfeld, Hinterzarten

Otto Ploeger, Bonn

Konrad v. Rabenau, Naumburg
Eugen Ludwig Rapp, Mainz

Friedrich Rau, Berlin

Martin Redeker, Kiel

Leopold Reidemeister, Berlin

Wolfgang Reimann, Rottach-Egern

Erwin Reisner, Berlin

Richard Reitzenstein, Göttingen

Rolf Rendtorff, Berlin

Trutz Rendtorff, Münster

Karl-Heinrich Rengstorf, Münster

Liselotte Richter, Berlin

Joachim Rogge, Berlin

Wilhelm von Rhoden, Naumburg

Karl Rose, Berlin

Leonhard Rost, Erlangen

Joachim Rothert, Bonn

Wilhelm Rudolph, Münster

Horst Seebaß, Bethel

Curt Seibert, Berlin

Martin Seils, Naumburg

Karl Silex, Berlin

Rudolf Smend, Göttingen

Oskar Söhngen, Berlin

Ernst Sommerlath, Leipzig

Franz Suchan, Berlin

Wolfgang Sucker, Mainz

Hans-Werner Surkau, Bonn

Kurt Scharf, Berlin

Ernst Schlee, Schleswig

Edmund Schlink, Heidelberg

Werner Schmauch, Greifswald

Kurt-Dietrich Schmidt, Hamburg

Martin Schmidt, Mainz

Paul und M. Schmidt, Eßlingen

Wieland Schmidt, Berlin

Wolfgang Schmieder, Frankfurt

Walter Schmithals, Marburg

Arnold Schmitz, Mainz

Hans-Peter Schmitz, Berlin

Friedrich Schnapp, Hamburg

Wilhelm Schneemelcher, Bonn

Max Schneider, Halle

Michael Schneider, Berlin

Hermann Schnitzler, Köln

Joachim Schoeps, Erlangen

Hans Scholz, Berlin

Erdmann Schott, Halle

Friedrich Schröter, Berlin

Adalbert Schütz, Bethel

Werner Schütz, Münster 
Wilhelm Schuster, Berlin Martin Schutzka, Berlin Luise von Schwartzkoppen, Berlin Wolfgang Schweitzer, Bethel Gustav Stählin, Mainz

Gerhard Stammler, Naumburg Ethelbert Stauffer, Erlangen Heinz Steinberg, Berlin Heinrich Steitz, Mainz Willy Stiewe, Berlin Rudolf Stöwesand, Berlin Hermann Strathmann, Erlangen August Strobel, Erlangen Rainald Stromeyer, Berlin Hans H. Studkenschmidt, Berlin Robert Stupperich, Münster

Kurt Tautz, Göttingen Jochen Thiele, Bredenbeck/Deister Martin Thilo, Berlin Oskar Thulin, Leipzig Joachim Tiburtius, Berlin Hermann Tiemann, Hamburg Juliane Trendelenburg, Stodkholm Wolfgang Trillhaas, Göttingen

Walter Uhsadel, Tübingen Wolfgang Ullmann, Naumburg Hans Urner, Halle

Dieter Vetter, Berlin Philipp Vielhauer, Bonn Friedrich Christian Viering, Berlin
Wilhelm Virneisel, Tübingen

Karl Vötterle, Kassel

Gotthard Vogel, Berlin

Heinrich Vogel, Berlin

Gottfried Voigt, Leipzig

Adalbert Voretzsch, Erlangen

Peter Wackernagel, Berlin

Stephan Waetzoldt, Berlin

Heinz Wagner, Leipzig

Gerhard Wallis, Halle

Otto Weber, Göttingen

Hans Wegener, Bremen

Carl Wehner, Heidelberg

Konrad Weiß, Rostock

Heinz-Dieter Wendland, Münster

Claus Westermann, Heidelberg

Kurt Wiesner, Leipzig

Werner Wiesner, Mainz

Ulrich Wildkens, Berlin

Ernst Wilm, Bielefeld

Wilhelm Witte, Marburg

Martin Wittenberg, Neuendettelsau

Ernst Wolf, Göttingen

Christoph Wolff, Erlangen

Hans Walter Wolf, Mainz

Hans-Georg Wormit, Berlin

Winfried Zeller, Marburg Walther Zimmerli, Göttingen

Franz Zimmermann, Berlin Hans Zopf, Berlin 